\title{
On research culture and mental health
}

\author{
Donata landolo and Gonçalo Silva on the need for an open discussion on mental health in academia.
}

( nce seen as a taboo topic of conversation, mental health issues within the academic world are now being more openly discussed. However, the stigma associated with coming forward, and the many interlocking factors affecting researchers' wellbeing, still persist. We need to work together on several elements of academic life for a real change to happen.

Short-term contracts and the fetishization of long work hours and productivity over both the quality of the science and the scientist's wellbeing have become hallmarks of the system as we know it ${ }^{1}$. Yet pursuing 'productivity' at all costs runs contrary to what science really needs: "less research, better research, and research done for the right reasons", as effectively penned in a 25 -year-old editorial that also nicely represents today's situation ${ }^{2}$.

The possibility of travelling, living and working in different countries and fields of research is exciting but can also leave scientists feeling insecure and unrooted. Security is needed when things don't go well, when results are not forthcoming, and when motivation and mood dip. The 'niche' that we build for ourselves, our network and our upbringing will make the difference in how we respond to challenging circumstances within and outside academia. This is where institutions should step in, by providing a safety net for the scientists when they need it the most.

In collaboration with the Marie Curie Alumni Association (MCAA)-UK Chapter and the Office of Postdoctoral Affairs of the University of Cambridge, we organized an event on mental health within academia, titled 'An open and frank discussion about mental health in an academic setting ${ }^{3}$. We asked representatives of funding bodies, principal investigators (PIs), early career researchers, psychiatrists and therapists to join the conversation. A physical theatre performer also helped us get in contact with each other and establish a safe environment where everyone could freely exchange personal experiences.

The awareness of the intrinsic diversity of the academic community is one of the themes that came out of the event. There are millions of us, with different backgrounds, identities and life trajectories. No one solution will work for everyone any more than a single drug will cure every ailment.

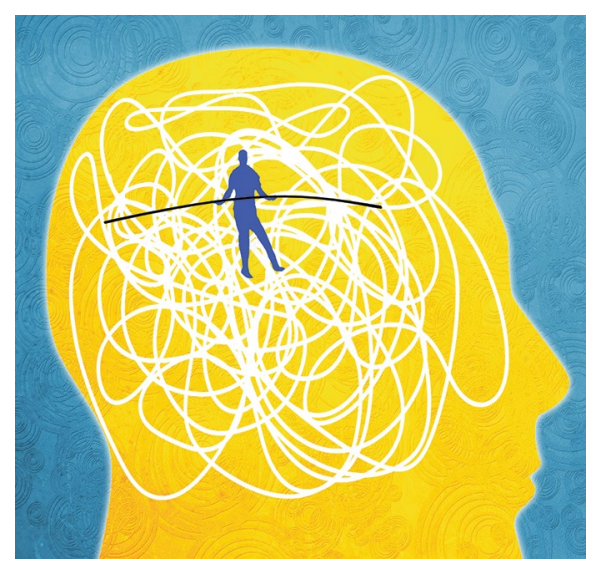

Credit: Lorenzo Rossi/Alamy Stock Photo

Loneliness, isolation, fear of failing and depression appeared to be very common experiences in our audience, as they seem to be in most of academia. Embracing resilience, or better 'antifragility' - the ability to harness stressing factors in order to improve one's capacity to thrive - was suggested by John Tregoning, as an approach to empower ourselves by harnessing the challenges we live through ${ }^{4,5}$.

We shared some positive examples, such as the counselling services on demand provided by the University of Cambridge and some of its constituent colleges. However, a huge disparity emerged in terms of access to support, among different UK institutions but also between countries. Given the intrinsic mobility of the researcher's job, initiatives at a European or even global level to create a network of support available in any country we live could certainly be beneficial. A positive step in this direction is represented by online counselling services for expats, such as those offered by the associations AIRI (https:// www.airicerca.org/english-summary) and RUMO (https://www.rumo.solutions), which collaborated with our event.

The main solutions that were put forward were to provide adequate training for PIs in team management skills, to value researchers rather than just PIs - as research has always been a collaborative effort - and to welcome the wide diversity of our community. Further measures, from improving the transparency of academic career progression to simply making the work environment more welcoming, were also discussed. We also feel there is the need to adopt a different model of evaluation, to bridge the divide between the reality of the scientific community and the current priorities for academic career progression.

Encouragingly, the MCAA and EURODOC have recently released a joint call for sustainable research careers ${ }^{6}$, asking for a long-awaited evolution of the academic system. However, it would be disingenuous for us to suggest that this could happen overnight. And yet, all signs point to change being on the horizon. Student-led initiatives are popping up in even the most corporatized of universities, senior scientists are beginning to open up about their mental health ${ }^{7}$, and major organizations like the Wellcome Trust and the Royal Society ${ }^{8}$ are discussing changes in their funding policies and fostering dialogue among all the key players. It is only through collaboration that a credible solution to such a complex problem can be found.

We believe that academic institutions, such as universities, funding bodies and publishers, have a moral imperative to help us catalyse change in this broken research culture, no matter how laborious such a process might be.

\section{Donata landolo (iD) 1,2 and Gonçalo Silva (D) 3} ${ }^{1}$ Mines Saint-Etienne, University of Lyon, Université Jean Monnet, INSERM, Saint-Etienne, France.

${ }^{2}$ Department of Chemical Engineering and Biotechnology, University of Cambridge, Cambridge, UK. ${ }^{3}$ School of Biological Sciences, Queen's University Belfast, Belfast, UK.

e-mail:donata.iandolo@emse.fr;

grosasdasilva01@qub.ac.uk

Published online: 22 July 2019

https://doi.org/10.1038/s41563-019-0441-1

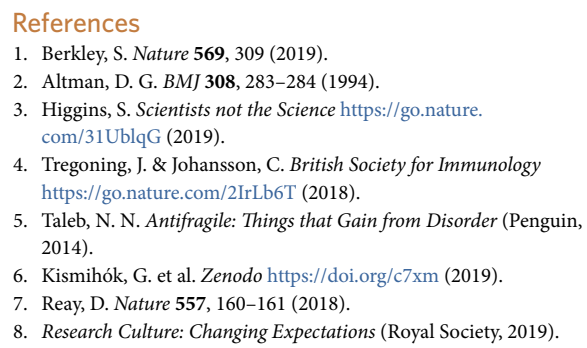

We acknowledge S. Higgins for his insightful comments on the article. We also thank M. L. Greco, the MCAA-UK chapter, the OPdA of the University of Cambridge for making the workshop possible, and the attendees and speakers for their invaluable contributions. 